irritant action as a cause of fibrous hyperplasia are much more forcible or convincing. Indeed, if by irritant action is meant hypermaximal stimulation-i.e., disease-producing stimulation-it cannot be directly a cause of growth. Now, does the action of the supposed irritant affect the functioning hepatic cells, or does it affect the connectivetissue cells of the liver. If it is to be a cause of growth it must affect primarily and directly the functioning cells which a priori would seem the more likely to respond to this form of stimulus. Yet if hepatic cirrhosis were due to the indirect action of an irritant the process should be in evidence at a much earlier period in disorders of the liver than is found to be the case. lf, therefore, the operation of these influences can be excluded or rendered doubtful, and if it can be shown, as I have attempted to do, that fibrosis depends in some instances solely on increased nutrition, it is not unreasonable to suppose that hepatic cirrhosis may be due to a similar cause.

The pathogenesis of hepatic cirrhosis advanced here is that the functioning cells of the liver undergo chronic hyperstimulation from overwork by means of excessive food, alcohol, and so forth, and in course of time the functioning protoplasm becomes unable to respond to the demands made upon it. This entails protoplasmic insufficiency and degeneration, and the consequent interference with function begets inability to control adequately cell nutrition and food supply, which leads to an increased nutritive stimulation of the fibrous tissue cells" and thereby to hyperplasia of these elements. It follows, as in the instances cited above, that under the operation of the morbid process hypothesised here, liver cell insufficiency and degeneration go hand-in-hand with the hepatic cirrhosis, but it is quite conceivable that a considerable degree of fibrous hyperplasia might be observed before any microscopical evidence of protoplasmic degeneration of liver-cells could be found; indeed, in this connexion it may be unhesitatingly affirmed that physiological-i.e., functionalinadequacy may obtain without any discoverable anatomical change. In this particular, at all events. the theory does not clash with practice.

Cirrhosis of the liver can be produced experimentally. If one of the hepatic ducts be ligatured the area drained by the duct that is obstructed undergoes cirrhosis. Here there is no place for inflammatory influences or for the action of an irritant. On the other hand, it may reasonably be supposed that the liver-cells are put out of action, and their functions being in abeyance the fibrous tissue cells grow under the increased and unaccustomed stimulus of uncontrolled nutrition. A similar condition may be observed in the case of the pancreas and salivary glands when the excretory ducts of these organs become blocked by stone or by stricture, \&c.

The pathogenesis of fibrous hyperplasia advanced here applies equally well to the explanation of the mode of production of renal cirrhosis. Thus, in consequence of the overwork that is occasioned by long-continued hyper-stimulation from various causes, the renal cells undergo degenerative changes, and the resulting loss of control over glandular nutrition affords the opportunity for increased growth to the fibrous tissue cells of the kidney which they take advantage of

The tibrosis of mechanical congestion.-The pathogenesis of the fibrosis that accompanies venous congestion and lymphatic obstruction is not difficult of explanation if the theory that has been advanced in this paper be well founded. It is necessary only to point out that in consequence of the altered circulatory conditions the nutrition of the more highly specialised cells of the organ involved, say, for instance, the liver, is imperfectly performed. The interference with cell nutrition which is not infrequently evidenced by protoplasmic degenerative changes implies more or less disturbance and loss of control over food supply, so that, even under the altered circulatory conditions, there is a relatively increased nutritional stimulation of the fibrous tissue cells, which therefore proliferate.

Senile fibrosis. - From infancy to old age there is a tendency, for reasons which in the light of the views advanced here are obvious, to a gradual increase in the amount of fibrous tissue throughout the body; and herein extremes meet, since premature fibrosis means premature senility. The liability to the growth of fibrous tissue throughout the body increases after middle life, and the rate of increase is regulated for the most part by the degree of functional capacity that is possessed and displayed by the more highly specialised tissne cells. During and after middle life the functional capacity of the more highly specialised cells diminishes throughout the body, yet how often does it not happen that a man of between 50 and 60 years of age retains the table habits of his youth. It comes to pass sooner or later that the limits of the declining functional capacity of the more highly-specialised cells, more particularly of such organs as the liver and kidneys, which deal primarily and principally with the elaboration of food and the elimination of waste products, are exceeded, and the consequent breakdown of protoplasmic efficiency shows itself morphologically by degenerative changes and functionally by imperfect metabolism, and, according to the views expressed here, by deficient control over the food-supply to the more lowly organised tissues. 'The nutrition of the fibrous tissue in the organs that are implicated is now imperfectly restrained and a gradually increasing fibrosis ensues. Herein, too, it may be observed, lies one explanation at least of the occurrence of obesity in elderly people. The brain, spinal cord, heart, liver, and kidneys are the organs chiefly affected in senile fibrosis These observations have, moreover, an important practical bearing on the necessity for the adoption of abstemious food habits after middle age, more especially in persons of sedentary habit. If the arguments brought forward in this paper carry conviction it would appear that the essential and fundamental element in the pathogenesis of fibrous hyperplasia is increased nutrition consequent on the partial or complete loss of the control that is normally exercised over the nutritive supplies to the fibrous tissue by the more highly specialised cells with which it is associated.

Throughout this paper the question of the influence of the nervous system has been carefully excluded, inasmuch as the operation of this factor on the incidence of fibrosis, though undetermined, is probably the same in all cases. Nevertheless, there is another side of the question with respect to the influence of innervation that must be briefly considered. So far, at least, as the more highly specialised tissues are concerned, two distinct processes of cell life must be clearly distinguished-viz., the functioning and the vegetative. There can be little doubt that nervous connexions and nervous influences are much more closely associated with the functioning than with the purely vegetative side of cell life. Now, with the decline of functional capacity there is no doubt, a diminution of nervous influence, so that it would come about that, with respect to their claim on nutritive supplies, the degraded functioning cell in its vegetative condition would be pitted against the purely vegetative connective-tissue cell. It is clear that the degraded functioning cell could not respond by growth to a relatively increased nutritional stimulus, since it has lost the habit and power of proliferation. The vegetative connectivetissue cell, on the other hand, retains the habit and power of reproduction, and under the influence of the relatively increased nutritive stimulus responds by growth.

The further pursuit of this interesting side of the subject does not come within the scope of this paper, but it may be suggested that it is not improbable that from this aspect of the mode of origin of tissue hyperplasia the pathogenesis of new growths will ultimately be elucidated.

Upper Berkeley-street, W.

\section{ACUTE EMPHYSEMATOUS GANGRENE.}

\section{By N. H. CHOKSY, M.D. Hon. Caus. Freib.,} SPECIAL ASSISTANT HEALTH OFFICER IN CHARGE OF ARTHUR-ROAD HOSPITAL, BOMBAY.

THIS affection, which has been variously designated by different observers but is usually known as "emphysematous gangrene," is of infrequent occurrence and very few records of cases are to be found in medical literature. Considering, however, the gravity of its nature, progress, and consequences once the infection has begun, and that at the same time it is amenable to treatment if it is diagnosed early and if suitable measures are applied, a thorough knowledge of its onset, course, symptoms, and treatment is absolutely necessary. With this view I propose to refer to four cases that came under my observation at the Arthur-road Hospital, Bombay, during the course of the last two years. 
The wost recent contributions on the subject are those of Mr. E. M. Corner and Dr. H. D. Singer,' and Dr. Welch.' The former describe a case under their care at St. Thomas's Hospital and discuss the bacteriology of the disease at length. They believe that the specific micro-organism of the disease exists in two forms, acrobic and anaerobic, the first being generally associated with streptococcus pyogenes and staphylococcus and the second occurring in pure cultures. Three varieties of the latter are mentioned-viz. (1) bacillus emphysematosus (Fraenkel), probably identical with bacillus perfringens (Veillon and Zuber); (2) bacillus cedematis maligni (vibrion septique Pasteur) ; and (3) bacillus aerogenes capsulatus (Welch and Nuttall). Mr. Corner and Dr. Singer maintain that the bacillus remains local in its action, is non-pathogenic to undamaged tissues, and infects only such as have become less resistant either by trauma or injection of bacterial toxins. In his Shattuck Lecture on the Morbid Conditions caused by the Bacillus Aerogenes Capsulatus, delivered before the Massachusetts Medical Society, Dr. Welch presents us with an almost classical summary of his own researches and those of other observers, and shows how wide and varied may be the diffusion of the germ and its effects when invading the various tissues of the body. For the bacillus does not confine its attentions to the external tissues of the body alone, but it may infect the genito-urinary tract, the gastrointestinal canal, the peritoneum, and even the large internal organs like the liver, lungs, \&c. Dr. Welch says that by far the most common specific cause of emphysematous gangrene is the bacillus aerogenes capsulatus and that numerous organisms, described by other observers under different names, are to all intents and purposes either identical with it or that at least one of them is an aerobic bacillus, probıbly identical with Sanfelice's bacillus pseudo-cedematis maligni, which also is capable of producing this affection, but with much less frequency than the bacillus aerogenes capsu latus. Welch and Nuttall's observations with regard to its wide distribution in nature have received confirmation at the hands of others and the natural habitat of the bacillus has been determined to be in the intestinal canal and the soil, and as it is eliminated in the frces the possibility of its being found upon the human skin has also been recognised. Only 46 cases of emphysematous gangrene, in all of which the bacillus had been demonstrated, occur in recent medical literature, and Dr. Welch gives a complete analysis of these. In all but five of the 46 cases the gangrene followed traumatism or a surgical operation. There was compound fracture in 18 cases, bullet and gunshot wounds existed in seven, the condition supervened after infusion of salt solution in three. hypodermic injections in two, and in the rest it followed surgical operations or other injuries of varying degree.

It is not absolutely necessary that there should be extensive injuries to bring about the infection, and five cases have been recorded in which the gangrene followed upon hypodermic injections and infusion of normal salt solution. Even the most unirritating of solutions may give rise to it, and this occurs when the patient's vital forces have become greatly depressed by Asiatic cholera, typhoid fever, plague, surgical shock, post-partum hæmorrhage, \&c. There is, according to Dr. Welch, good reason to believe that the intact tissues of human beings in health possess marked resistance to the gas bacillus, and Muscatelle and Gangitano also say that it attacks tissues already altered in their vitality by injury, by other pathogenic micro-organisms, by toxins, or by other depressing factors. He does not, however, believe that under all circumstances it is incapable of attacking healthy tissues. Two forms of the gangrene have been described by Pirogoff-one a rapidly spreading form and the other more localised, and it is assumed by some that the form of the gangrene is determined by the fact of the infection with the gas bacillus being either pure or mixed with other pathogenic bacteria, and that in the latter event the form is progressive, and in the former slow and more or less localised. This, however, is not borne out by Dr. Welch, as he has observed both the varieties in the unmixed pure infection, although it is not always possible sharply to define them clinically The bacillus aerogenes capsulatus causes not only emphysematous gangrene, but also gaseous phlegmon or abscesses and it may infect any tissues or organs, even the brain not excepted. After citing a number of cases Dr. Welch con- cludes that the bacillus aerogenes capsulatus in combination usually with pyogenic bacteria is the chief, if not the sole, cause of the gaseous abscesses or emphysematous gangrene which occasionally follow hypodermic injections. It appears that the fluids injected were not examined, but he thinks that the bacillus was either in them or in the syringe, whilst the fact that it might have been conveyed from the skin of the patients, or perhaps it may have reached the injured tissues from the intestine through the circulation, should not be overlooked.

The cases at the Arthur-road Hospital, Bombay, resolve themselves into two groups The first group comprised three cases of bubonic plague under the specific treatmentviz., with Professor Lustig's curative serum-during March, 1900 , when the fourth epidemic of plague was at its height. In the second there was only one case-viz., that of relapsing fever, and it occurred in April, 1901

Emphysematous gangrene-or, to speak more correctly, emphysematous and gaugrenous abscess-developed almost simultaneously in the plague cases at the site of the serum injections, and although about 1150 cases have been treated with the serum within the last four years no such mishap has been observed in any except the three above mentioned. The procedure followed in injecting the serum was as follows. The skin was first thoroughly cleaned with lysol solution, then with ether, and lastly with sublimate solution ( 1 in 1000). The syringe and needles were sterilised by boiling in 2 per cent. carbolic lotion every morning and were then kept im mersed in 5 per cent. carbolic solution. Two or three needles were usually employed for the purpose, and whenever possible the same needle was not used in succession. On account, however, of the paucity of needles obtainable locally and the number of injections made daily-about 15 to 20 morning and evening-together with 1 he want of skilled assistance, it was not possible to sterilise every needle after each injection. As soon as one was withdrawn it was wiped with cottonwool soaked in 5 per cent. carbolic lotion and was immediately immersed in the same lotion for some minutes until it was required for a subsequent case. The injections were almost invariably made subcutaneously on the inner and outer aspects of the thigh alternately and sometimes on the arms, and the quantity injected each time varied from 20 to 40 or even 50 cubic centimetres. As soon as the first case was observed extra precautions were taken to insure a thorough aseptic condition of the syringes and needles, but in spite of this a second and then a third case developed. Ultimately the syringes and the needles in use were discarded and a new set was substituted. Bacteriological examination of the serum used showed it to be sterile, and as the syringes and needles were boiled daily, and at that time twice daily, it is difficult to realise how infection could have originated from them in three cases only, whereas from 15 to 20 were being injected twice daily with the same appliances. It is probable that infection may have spread from the first patient, whose skin was extremely tough and required some amount of force to drive the needle in, and that the same needle might have conveyed the infection to the other cases on the same day, but that the gangrene developed in proportion to the resistance offered by their tissues. The gangrene occurred in, and was localised to, the thigh only, involving almost the whole of its front aspect, and was preceded by signs of acute inflammation, the skin looking angry and codematous, and there was emphysematous crackling. The systemic reaction was also great and the patients got distinctly worse after these symptoms supervened. When the abscesses were opened fotid gas escaped in bubbles, together with unhealthy pus and sloughs of a dark and gangrenous colour. The muscles of the front of the thigh became gangrenous and pus and gas burrowed between them; large pieces of gangrenous muscle tissue were daily removed. The patients became steadily worse and succumbed, and there is not the least doubt that their deaths were hastened by this infection. The first case was that of a fairly strong and well-built man, but the two other patients were in an extremely weak and debilitated condition and were probably half-starved. Thorough bacteriological examination of the contents of the abscesses or of the necrosed tissue was not possible on account of pressure of work and the examination of the serum. Syringes and the needles gave negative results. It must be admitted that the grave nature of the cases was not immediately recognised, and it is probable that had an early opening been made and had the parts been appropriately treated the results' would have been 
different, as shown by the following case. This patient was suffering from relapsing fever and was receiving stimulants and hypodermic injections for cardiac failure. The injections contained a combination of caffeine, spartein, strychnine, and atropin, made up in rum, and the injections were repeated every six hours. They were generally given on the arm in doses of 20 minims. About six days after receiving them the patient complained of a swelling on the left arm which on examination left no doubt that we had to deal with a gaseous abscess. The patient was immediately isolated, a free incision was made in the arm, and gaseous pus with sloughs of a dark gangrenous colour escaped. The parts were thoroughly irrigated with sublimate lotion and dressed with iodoform, and this was repeated three times a day. Under this treatment there was a decided improvement and within three days the parts assumed a healthy appear. ance and healed gradually without any trouble. 'This abscess was strictly localised to the front of the arm and involved in its destruction a part of the biceps. The fluid injected was examined bacteriologically but with negative results, and Dr. N. Berestneff of the Moscow University kindly undertook the bacteriological examination of the contents of the abscess. He was able to obtain an aerobic growth, but was of opinion that it was not Fraenkel's bacillus. It may have been Sanfelice's bacillus pseudocedematis maligni refenred to by Dr. Welch. This patient was well-built, but had an extremely thick skin which was difficult to penetrate with a hypodermic syringe needle. Although several patients were receiving the same injection of the same fluid and by the same syringe at that time no other casts developed.

The above cases confirm the statements of Dr. Welch and others, that tissues damaged by the injection of bacterial toxins are prone to infection by the gas bacillus, and that even the most unirritating solutions injected hypodermically are at times followed by such infection. Hypodermic injections of cardiac stimulants form by far the most important feature of plague treatment, as well as that of relapsing fever and cholera, \&c., and although thousands of injections have been made within the last five years it was in one case only, that of relapsing fever, that gaseous abscesses supervened. A thorough consideration of all the facts bearing upon these cases tends to show that the infection must have been conveyed by the needles from the skin of the patients. Last year during the prevalence of cholera the same injections were resorted to very freely and yet in not a single instance was there an infection of a similar nature although small localised abscesses or blisters are by no means uncommon in these cases on account of the sluggishness of the circulation.

Bombay.

\section{A FAMILY OF THREE CASES OF THE PERONEAL TYPE OF MUSCULAR} ATROPHY (CHARCOT-MARIETOOTH-HOFFMANN).

By W. B. WARRINGTON, M.D., M.R.C.P. LOND., PHYSICIAN TO THE DAVID LEWIS NORTHERN HOSPITAL; DEMONSTRATOR OF PATHOLOGY IN" THE UNIVERSITY COLLEGE, LIVERPOOL.

With a Note on the Result of Surgical Treatment BY ROBERT JONES, F.R.C.S. EDIN.,

HONORARY SUREEON, ROYAL SOUTHERN HOSPITAL, LIVERPOOL.

THE cases here recorded are examples of a comparatively rare type of disease the position of which amongst the muscle atrophies is still obscure, though two recent examinations made by the present-day methods give some indications of its pathology. The ordinary type of progressive muscular atrophy as described by Aran and Duchenne usually commences in the small muscles of the hand. Hammond mentions that when the legs are first affected the condition is often hereditary. The distinct features of such an hereditary type of peroneal atrophy were, however, first clearly indicated by Howard Tooth ${ }^{2}$ and Charcot and Marie ${ }^{2}$ in 1886. Hoffmann ${ }^{3}$ in 1889 and 1891 exhaustively discussed

1 The Peroneal Type of Progressive Muscular Atrophy; Cambridge University Thesis, 1886 ; St. Bartholomew's Hospital Reports, vol. xxv. 2 Revue de Médecine, 1886.

A Archiv fur Psychologie und Nervenkrankheiten, xx.; Deutsche Zeitschrift für Nervenheilkunde, 1891, p. 95. the subject, adopting a distinct pathology for this type of disease, as is shown by the title of his papers ("Ueber Pro. gressive Neurotische Mi uskelatrophie"). A further important communication was made in 1893 by Bernhardt ${ }^{4}$ and in 1899 Sainton ${ }^{5}$ gave the results of a thorough microscopical examination of the nervous system in a case of this type. Other records of this and allied forms of atrophy have been published and have adde 1 considerably to our knowledge.

CASE 1 (Fig. 1).--The patient was a healthy-looking

Fig. 1.

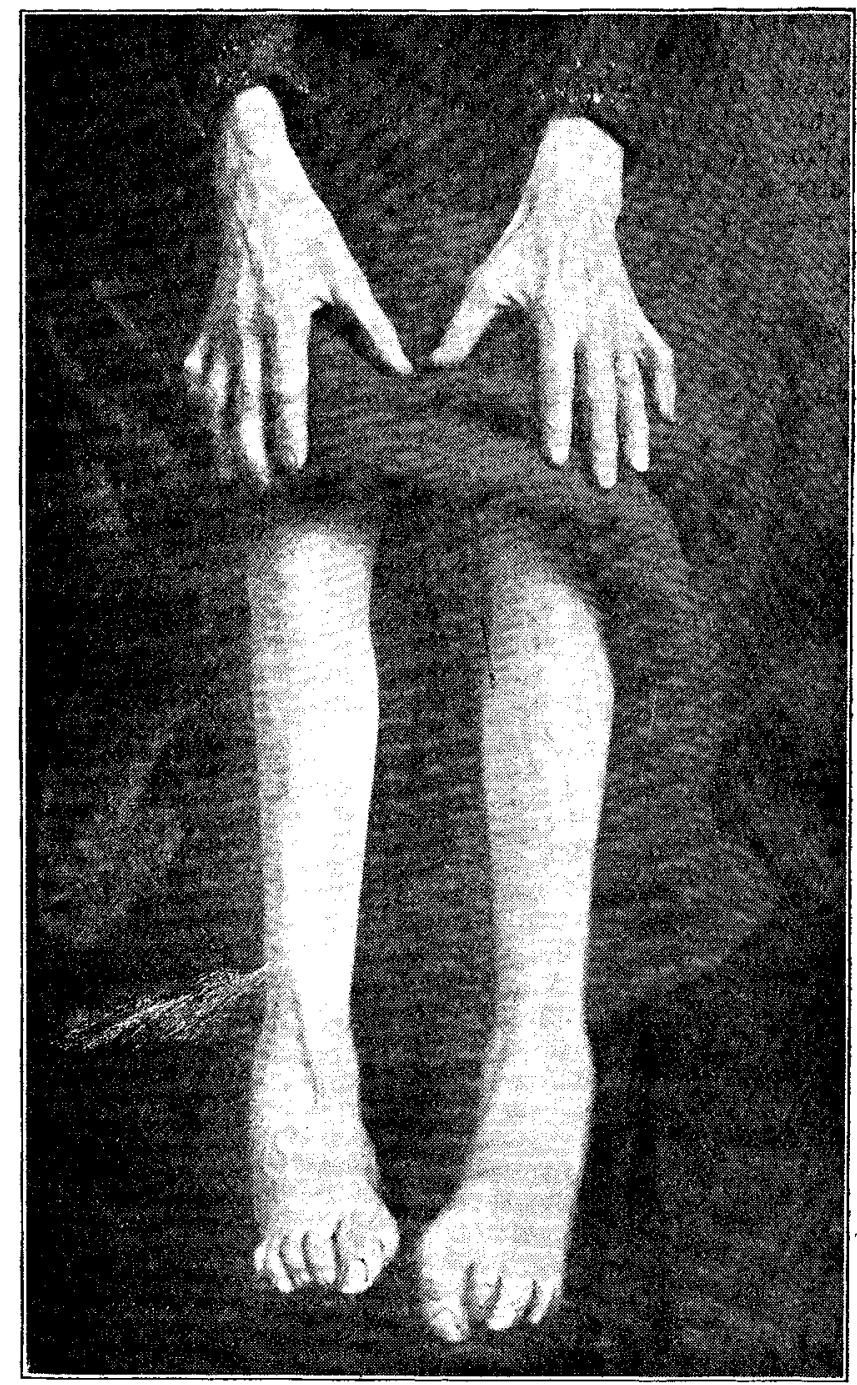

Case 1. Showing wasting of first dorsal interossei and slight club-foot. country woman, aged 56 years, practically free from any disability due to the condition observed. Both her father and mother lived to an old age and she was not acquainted with any relative who had suffered in a similar manner. She had borne five children, the two eldest and the fourth being girls; they are at present alive and in good health. The third and fifth were boys and are the subjects of the present note. The patient herself had always had good health and her pregnancies had been natural. She stated that when about seven years old she found that her ankles, especially the right, easily "turned in" and that consequently she often suffered from sprains. She was unaware that there was anything unusual about her hands. With regard to her present state, the muscles of the thenar eminence and of the first interosseous space were wasted in both hands, and also, but to a less extent, the hypothenar eminences. The other interossei muscles appeared to be normally developed. This condition appeared to cause very little disability, as with the exception of the finer movements of the thumb and forefinger there was no paralysis. The muscles of the rest of the upper extremity and of the shoulder girdle did not appear to be in any way affected. In the lower extremity deformity was more advanced and unequally developed on either side. On the right the foot was hollowed and inverted and also somewhat dropped. The tendon of the tibialis anticus stood ont like a taut cord. The toes and ankle-joint could be freely moved in all directions except that of eversion owing to complete 